\title{
EMPIRICAL RESULTS ESTABLISHING THE THERMAL INDEPENDENCE OF THE GRAIN-BOUNDARY REFLECTION COEFFICIENT
}

\author{
C. R. PICHARD, A. J. TOSSER and C. R. TELLIER \\ Laboratoire d'Electronique, Université de Nancy-I, C.O. 140, 54037-Nancy-Cedex France
}

(Received October 1, 1979)

\begin{abstract}
Starting from the effective Fuchs--Sondheimer model of electronic conduction in thin polycrystalline films and implementing the asymptotic expressions of conductivity it is shown that the hypothesis of temperature independence of the grain boundary reflection coefficient agrees with previously published results.
\end{abstract}

\section{THERMAL EXPANSION OF THE GRAIN- BOUNDARY REFLECTION COEFFICIENT}

Theoretical studies have shown ${ }^{1}$ that the expression for the electronic conductivity of a thin metallic film in the Mayadas-Shatzkes model (M. S. model) ${ }^{2}$ may be replaced by an expression derived from the Fuchs-Sondheimer model (F. S. model), ${ }^{3}$ in which the bulk conductivity is replaced by the conductivity of an infinitely thick polycrystalline film (the grain-boundary resistivity) and the relative thickness is replaced by the grain-boundary relative thickness, i.e. the relative thickness divided by the M. S. function $f(\alpha)$ which characterizes the effect of grain-boundary scattering. An effective mean free path had been defined ${ }^{1}$ for this purpose.

Theoretical expressions of the temperature coefficient of resistivity can be deduced from the expressions of the conductivity, ${ }^{1,4,5}$ assuming that thermal variations in film thickness, grain size and reflection coefficient $r$ are negligible.

Numerical results ${ }^{1,5}$ related to theoretical formulae have shown that M. S. expressions and modified F. S. expressions coincide within an accuracy of $5 \%$.

Hence the polycrystalline (monocrystalline) film can be considered, from a theoretical point of view, as built in an imaginary isotropic material whose bulk imaginary resistivity is equal to that of an infinitely thick polycrystalline (monocrystalline) film and in which electrons are scattered at the external surfaces. The conduction properties are then described by the following equations ${ }^{1}$ for polycrystalline films.

$$
\begin{aligned}
& \sigma_{f}=\sigma_{g}\left[1-A\left(k_{g}\right)\right] \\
& \beta_{f}=\beta_{g}\left[1+\frac{-A\left(k_{g}\right)+B\left(k_{g}\right)}{1-A\left(k_{g}\right)}\right]
\end{aligned}
$$

where

$\sigma_{f}$ is the film conductivity and $\beta_{f}$ its t.c.r.,

$\sigma_{g}$ is the grain-boundary conductivity and $\beta_{g}$ its t.c.r.,

$$
k_{g}=\frac{a}{l_{0} \cdot f(\alpha)}=\frac{k}{f(\alpha)}
$$

where $a$ is the film thickness, $l_{0}$ the electronic mean free path in the bulk material and $f(\alpha)$ is the M. S. function defined by: ${ }^{2}$

$$
\begin{aligned}
f(\alpha) & =1-\frac{3}{2} \alpha+3 \alpha^{2}-3 \alpha^{3} \ln \left(1+\frac{1}{\alpha}\right) \\
\alpha & =\frac{l_{0}}{a_{g}} \frac{r}{1-r}
\end{aligned}
$$

where $a_{g}$ is the average grain diameter and $r$ the electronic reflection coefficient on grain-boundary, and:

$$
\begin{aligned}
& A\left(k_{g}\right)=\frac{3}{2 k_{g}}(1-p) \int_{1}^{\infty}\left(\frac{1}{t^{3}}-\frac{1}{t^{5}}\right) \\
& \frac{1-\exp \left\{-k_{g} t\right\}}{1-p \exp \left\{-k_{g} t\right\}} \mathrm{d} t \\
& B\left(k_{g}\right)=\frac{3}{2}(1-p)^{2} \int_{1}^{\infty}\left(\frac{1}{t^{2}}-\frac{1}{t^{4}}\right) \\
& \frac{\exp \left(-k_{g} t\right)}{\left\{1-p \exp \left(-k_{g} t\right)\right\}^{2}} \mathrm{~d} t
\end{aligned}
$$


where $p$ is the fraction of electrons specularly scattered at the external surfaces.

In the F. S. model the corresponding equations are $:^{1,3,6}$

$$
\begin{aligned}
& \sigma_{f}=\sigma_{0}[1-A(k)] \\
& \beta_{f}=\beta_{0}\left[1+\frac{-A(k)+B(k)}{1-A(k)}\right]
\end{aligned}
$$

For $k>1$, approximate forms of Eqs. (8) and (9) are: ${ }^{3,7,8}$

$$
\begin{aligned}
& \sigma_{f} \approx \sigma_{0}\left[1-\frac{3(1-p)}{8 k}\right] \\
& \beta_{f} \approx \beta_{0}\left[1-\frac{3}{8} \frac{(1-p)}{k}\right]
\end{aligned}
$$

These expressions are valid for $k>1$, with an accuracy of $5 \%{ }^{8}$ It follows, from Eqs. (10) and (11):

$$
\rho_{f} \cdot \beta_{f} \cdot\left(\rho_{0} \cdot \beta_{0}\right)^{-1} \approx 1
$$

It may be noted that $\beta_{0}$ and $\beta_{f}$ (Eqs. (9) and (11)) were calculated ${ }^{6}$ by logarithmic differentiation of the conductivity equation with the assumption that thermal variation in geometrical parameters is negligible in relation to thermal variation in the mean free path.

Values of conductivity and t.c.r. (they are marked with an "exp" index in the equations) of sputtered $\mathrm{Al}$ and $\mathrm{Zn}$ polycrystalline films have been obtained experimentally, ${ }^{8-11}$ and it has been experimentally observed $^{6,9}$ that:

$$
\left(\rho_{f_{\text {exp }}} \cdot \beta_{f_{\text {exp }}}\right) \cdot\left(\rho_{g_{\text {exp }}} \cdot \beta_{g_{\text {exp }}}\right)^{-1} \approx 1
$$

in a large range of values of $k_{g}$ (Figure 1).

Since Eqs. (12) and (13) are identical experimental evidence is given for the validity of the assumption related to the negligible thermal variations in conduction parameters except in mean free path. Hence, thermal variations in reflection coefficient $r$ can also be neglected in relation to variations in mean free path in the range $k_{g}>1$, for the calculation of $\sigma_{f}$ and $\beta_{f}$.

\section{SOME CONSEQUENCES}

Consequently the t.c.r. of unsupported films can be easily deduced from the expression of film conductivity in the imaginary F. S. model






$$
\sigma_{f} \approx \sigma_{g}\left(1-\frac{3}{8} l_{g} \frac{1-p}{t}\right)
$$

with $l_{g}=l_{0} \cdot f(\alpha)$.

Since

$$
\beta_{g}=\frac{1}{l_{g}} \frac{\mathrm{d} l_{g}}{\mathrm{~d} T}
$$

where $T$ is temperature.

$$
\begin{aligned}
& \text { It yieldis: } \\
& \beta_{f p} \approx \beta_{g}\left(1-\frac{3}{8} l_{g} \frac{1-p}{t}\right)
\end{aligned}
$$

whenever $l_{g} \cdot t^{-1}<1$.

For monocrystalline films, Eqs. (1), (2), (14) and (15) are still valid if the $g$ index is related to infinitely thick monocrystalline film. Eq. (5) is also valid but the grain diameter is now equal to the film thickness and an asymptotic expression of Eq. (4) is then:

$$
f(\alpha) \approx 1-\frac{3}{2} \alpha=1-\frac{3}{2} \frac{l_{0}}{t} \frac{r}{1-r} \quad \alpha<1
$$

Hence:

$$
\frac{3}{8}(1-p) \frac{l_{g}}{t}=\frac{3(1-p)}{8} \frac{l_{0}}{t} f(\alpha) \approx \frac{3(1-p)}{8} \frac{l_{0}}{t}
$$

The relation between the t.c.r. $\beta_{g}$ of an infinitely thick film and the bulk t.c.r. is known: ${ }^{4}$

$$
\frac{\beta_{g}}{\beta_{0}}=1+\alpha \cdot \frac{\mathrm{d} f(\alpha)}{\mathrm{d} \alpha} \cdot[f(\alpha)]^{-1}
$$

For $\alpha<1$, an asymptotic expression of Eq. (18) is, from Eq. (4):

$$
\frac{\beta_{g}}{\beta_{0}} \approx 1-\frac{3}{2} \alpha
$$

Introducing Eq. (17) and (19) into Eq. (15) gives:

$$
\beta_{f m} \approx \beta_{0}\left[1-\frac{3}{2} \frac{l_{0}}{t} \frac{r}{1-r}-\frac{3}{8} \frac{l_{0}}{t}(1-p)\right]
$$

whenever $l_{0} \cdot t^{-1}<1$.

The validity of Eq. (15) has been previously shown by experiments related to polycrystalline films. ${ }^{9}$ Eq. (20) gives the literal form of a new approximate expression of the t.c.r. of monocrystalline films, whose theoretical validity has been recently established. ${ }^{12}$

Eq. (14) is also valid for monocrystalline films and can be transformed in the same way as Eq. (15).
It yields:

$$
\sigma_{f m} \approx \sigma_{0}\left[1-\frac{3}{2} \frac{l_{0}}{t} \frac{r}{1-r}-\frac{3}{8} \frac{l_{0}}{t}(1-p)\right]
$$

Eq. (21) agrees with the theoretical expression previously proposed by Thieme and Kirstein ${ }^{13}$ Eq. (12). It must be noted that more sophisticated approximate expressions must be used ${ }^{14}$ if a more extended range of validity is required.

All these consequences agree with previously published results and sustain the validity of the thermal independence of the grain-boundary reflection coefficient.

However, a more detailed analysis is necessary in the case of films firmly attached to a substrate since variations in the t.c.r. are due to thermal strains ${ }^{15}$ when there is a mismatch between thermal expansion coefficients of film and substrate. This point would be emphasized in a further paper.

\section{CONCLUSION}

The approximate thermal independence of reflection coefficient on grain-boundary is empirically established. It gives further physical validity to previous theoretical calculations related to conduction properties such as electric thermopower ${ }^{16}$ or thermal variations in the strain coefficient of resistivity ${ }^{17}$ or in the Hall coefficient. ${ }^{18}$

\section{REFERENCES}

1. C. R. Tellier, Thin Solid Films, 51 (1978), 311-317.

2. A. F. Mayadas and M. Shtazkes, Phys. Rev. B, 1 (1970), 1382-1389.

3. E. H. Sondheimer, Adv. in Physics, 1 (1952), 1-42.

4. C. R. Tellier and A. J. Tosser, Thin Solid Films, 44 (1977), 141-147.

5. C. R. Tellier and C. Boutrit, Thin Solid Films, 46 (1977), 307-313.

6. C. R. Tellier and A. J. Tosser, Rev. Phys. Appl., 13 (1978), 441-447.

7. K. L. Chopra, Thin Film Phenomena. (McGraw Hill, 1969).

8. C. R. Tellier and A. J. Tosser, Thin Solid Films, 43 (1977) $261-266$.

9. C. R. Tellier, A. J. Tosser and C. Boutrit, Thin Solid Films, 44 (1977), 201-208.

10. C. R. Tellier and A. J. Tosser, Le Vide, 189 S (1978), $25-31$.

11. C. R. Tellier; Vacuum, 28 (1978), 321-327.

12. C. R. Tellier, C. R. Pichard and A. J. Tosser, Thin Solid Films, 64 (1979), 1-2.

13. F. Thieme and W. Kirstein, Thin Solid Films, 30 (1975), $371-375$.

14. C. R. Tellier and A. J. Tosser,Electrocomp. Sci. Technol., 3 (1976), 165-169. 
15. P. M. Hall. Appl. Phys. Lett., 12 (1968), 212.

16. C. R. Tellier and A. J. Tosser, Thin Solid Films, 41 (1977), 161-166.
17. C. R. Tellier and A. J. Tosser, Electrocomp. Sci. Technol., 4 (1977), 9-17.

18. C. R. Tellier, C. R. Pichard and A. J. Tosser, Electrocomp. Sci. Technol., (in press). 

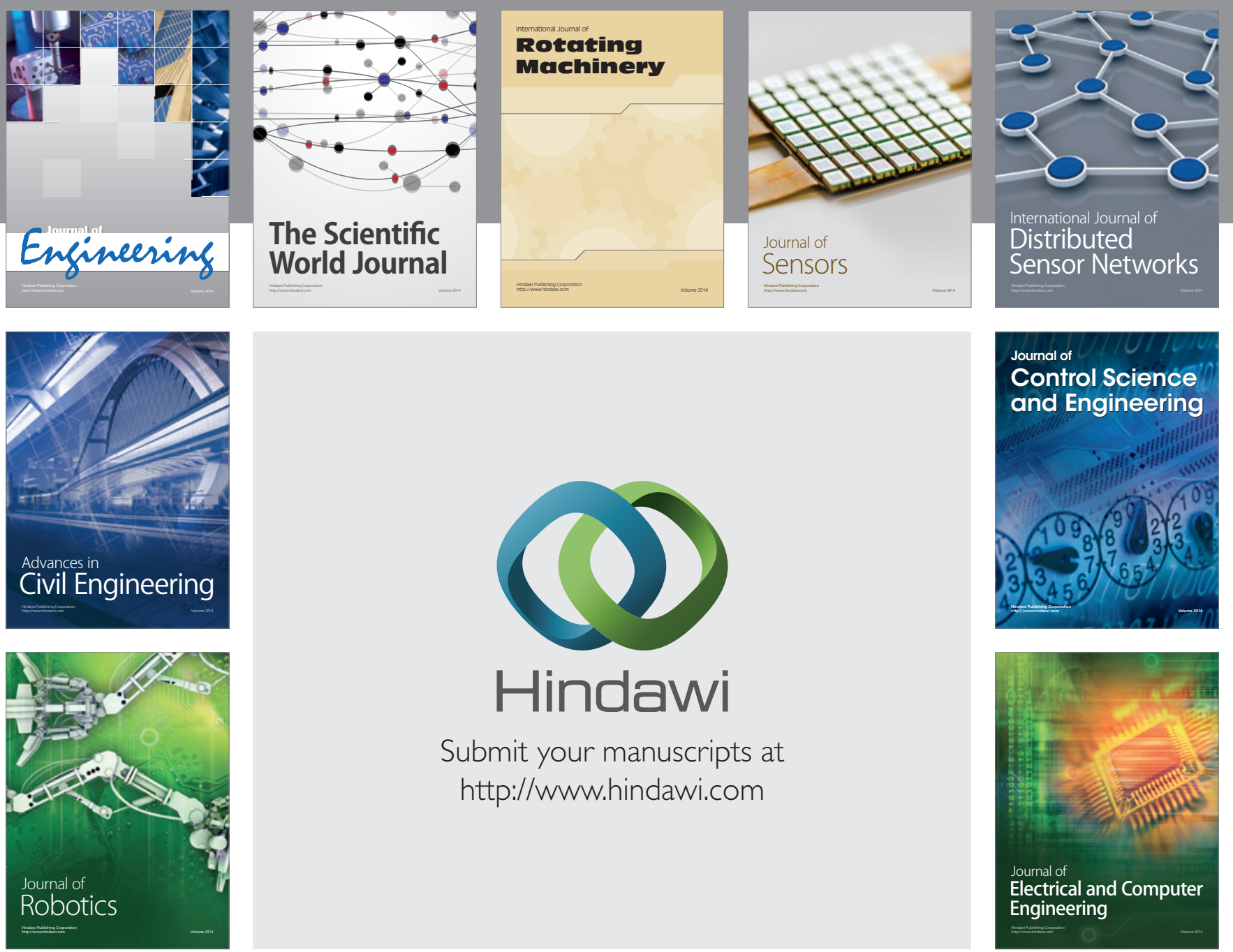

Submit your manuscripts at

http://www.hindawi.com
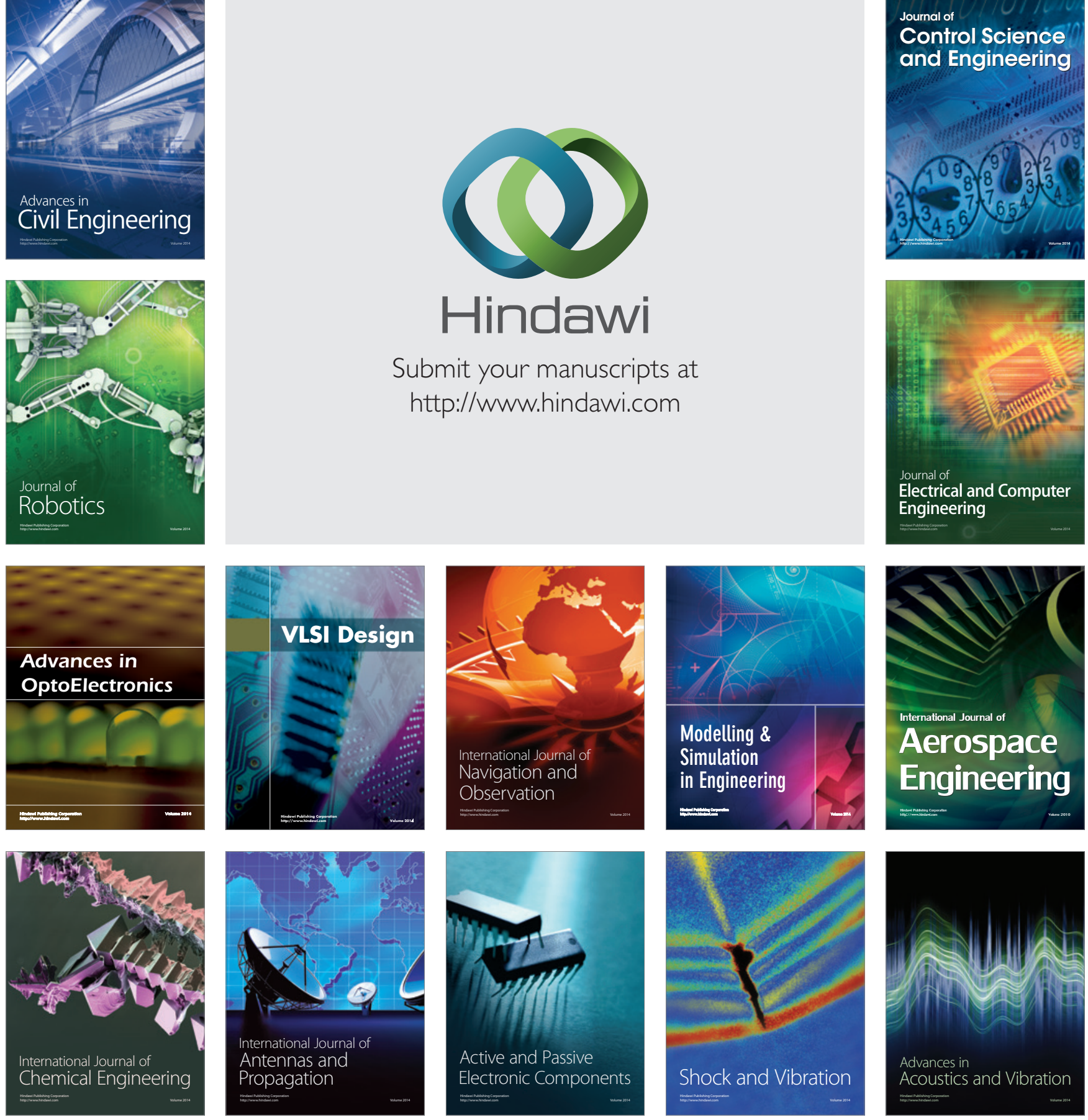